\title{
Nausea Subscale
}

National Cancer Institute

\section{Source}

National Cancer Institute. Nausea Subscale. NCI Thesaurus. Code C157703.

A subscale of the Pediatric Quality of Life Brain Tumor Module designed to assess issues associated with nausea. 